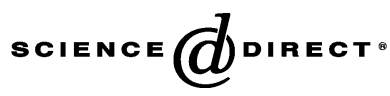

Economics Letters $\mathrm{xx}(2004) \mathrm{xxx}-\mathrm{xxx}$ economics

letters

www.elsevier.com/locate/econbase

\title{
Strategy-proofness and efficiency are incompatible in production economies
}

\author{
Justin Leroux* \\ Economics Department, Rice University, Houston, TX 77025, USA
}

Received 5 December 2003; accepted 7 April 2004

\begin{abstract}
In a production economy where a single private good is produced via a non-linear concave technology, no direct mechanism satisfies strategy-proofness and efficiency if the preference domain contains the class of linear preferences.
\end{abstract}

(C) 2004 Elsevier B.V. All rights reserved.

Keywords: Strategy-proofness; Efficiency; Production economies

JEL classification: D61; D71

\section{Introduction}

The trade-off between efficiency and strategy-proofness has been studied in great detail in the case of the distribution and exchange of private goods. These concepts together lead to decidedly unfair allocations of resources. Originating from a conjecture in Hurwicz (1972), this result was first proved in the two-agent case (e.g. Kato and Ohseto, 2002; Ju, 2003; Schummer, 1997; Sprumont, 1995; Zhou, 1991) examining various domain restrictions. Recently, Serizawa (2002) formally established this negative result for an arbitrary number of agents.

We study the same trade-off for simple production economies where a single private good is produced via a concave technology. Maniquet and Sprumont (1999) show that strategy-proofness and efficiency can coexist in a linear production model on the domain of classical economic preferences, even in

* Tel.: +1-832-778-0454; fax: +1-713-348-5278.

E-mail address: jleroux@rice.edu (J. Leroux). 
combination with anonymity. They call the resulting unique solution the equal budget free choice mechanism: every agent obtains the bundle she would choose if operating the technology alone.

We show that this positive result does not survive if the single technology is concave but not linear (though not necessarily strictly concave). Then strategy-proofness and efficiency are incompatible on the domain of linear preferences. Because strategy-proofness is a stronger property on larger domains, our result extends to any domain containing the class of linear preferences.

Shenker (1992) considers a cost-sharing model that includes ours as a special case. He states that if the technology exhibits decreasing returns to scale, any incentive compatible sharing rule is of the serial type. This would imply our result as serial-like methods are not first-best efficient. Yet, our result improves upon his statement on the strategy-proofness and efficiency trade-off in three respects. First of all, Shenker imposes smoothness conditions on the technology and on the allocation rule, which we do not. Secondly, his additional conditions on the technology amount in our setting to strict concavity of the production function, which we do not require. Lastly, and more importantly, his incentive compatibility criterion is much stronger than strategy-proofness: implementability in Nash equilibrium strategies is in fact even stronger than group strategy-proofness.

\section{The model and theorem}

Let $N=\{1, \ldots, n\}$ be the set of agents. Let $F$ be a strictly increasing, concave (though not necessarily strictly concave) non-linear function of $\mathbb{R}_{+}$to itself such that $F(0)=0$. A bundle is an element $z_{i}=\left(x_{i}, y_{i}\right) \in \mathbb{R}_{+} \times \mathbb{R}$, and an allocation is a list of $n$ bundles, $z=\left(z_{1}, \ldots, z_{n}\right)$, one for each agent. The set of feasible allocations is denoted by

$$
Z=\left\{z \in\left(\mathbb{R}_{+} \times \mathbb{R}\right)^{N} \mid \sum_{i} y_{i} \leq F\left(\sum_{i} x_{i}\right)\right\}
$$

For any subset $S \subseteq N$, we write $x_{s}=\sum_{i \in s} x_{i}$ and $y_{s}=\sum_{i \in s} y_{i}$.

Each agent is endowed with a preference, $R_{i}$, over $\mathbb{R}_{+} \times \mathbb{R}$ which is strictly monotonic: strictly increasing in $y_{i}$ and strictly decreasing in $x_{i}$. We denote by $\mathcal{R}_{0}$ the class of preferences. A preference profile is a list of $n$ preferences, $R=\left(R_{1}, \ldots, R_{n}\right)$. We sometimes write $R=\left(R_{j}, R_{-j}\right)$ for some $j \in N$. Let $\mathcal{L} \subset \mathcal{R}_{0}$ be the class of linear preferences. Each preference $L \in \mathcal{L}$ can be identified with a number $l \in \mathbb{R}_{++}$ that corresponds to the slope of its indifference curves in the $(x, y)$-plane. The corresponding utility for agent $i$ is $u_{i}\left(x_{i}, y_{i}\right)=y_{i}-l x_{i}$.

For any subset $A \subseteq \mathbb{R}_{+} \times \mathbb{R}$ and any preference relation $R_{i} \in \mathcal{R}$, we define $m\left(A, R_{i}\right)=\left\{z_{i} \in A \mid \forall z_{i}^{\prime}\right.$ $\left.\in A z_{i} R_{i} z_{i}^{\prime}\right\}$ to be the set of maximal elements of $A$ according to $R_{i}$. For any preference profile $R \in \mathcal{R}^{N}$, we denote by

$$
P E(R) \equiv\left\{z \in Z \mid \forall z^{\prime} \in Z\left[z_{i}^{\prime} R_{i} z_{i} \quad \forall i \in N \Rightarrow z_{i}^{\prime} I_{i} z_{i} \quad \forall i \in N\right]\right.
$$

the set of Pareto-efficient allocations. 
Let $\mathcal{R} \subseteq \mathcal{R}_{0}$, a direct allocation mechanism (or mechanism) $\mu: \mathcal{R}^{N} \rightarrow Z$ associates with each preference profile a feasible allocation. We are interested in the following axioms to be verified by a mechanism $\mu$ :

Pareto efficiency (PE) $\forall R \in \mathcal{R}^{N}, \mu(R) \in P E(R)$.

Strategy-proofness (SP) $\forall R \in \mathcal{R}^{N} \forall i \in N \forall R_{i}^{\prime} \in \mathcal{R}, \mu_{i}(R) R_{i} \mu_{i}\left(R_{i}^{\prime}, R_{-i}\right)$.

Theorem. Let $\mathcal{L} \subseteq \mathcal{R} \subseteq \mathcal{R}_{0}$. No mechanism $\mu: R^{N} \rightarrow Z$ satisfies $S P$ and $P E$.

As in Maniquet and Sprumont (1999) we determine the shape of the agents option sets, i.e. the sets of attainable bundles given the reports of others. We proceed by contradiction, assuming that a mechanism $\mu$ satisfies SP and PE to later show that the shapes of the option sets generated by $\mu$ are not feasible; namely, budget balance is violated.

We start the proof with two lemmas. Lemma 1 states a general property of option sets and can be found in Maniquet and Sprumont (1999). Loosely speaking, Lemma 2 states that if a function $f$ is concave and if a function $g$ has the same slope as $f$ for every value of $t$ in its domain, then $g$ coincides with $f$ up to a positive constant. First, a definition: a strictly increasing subset of $\mathbb{R}_{+} \times \mathbb{R}$ is a set $h \subset$ $\mathbb{R}_{+} \times \mathbb{R}$ such that for all $\left(x_{i}, y_{i}\right),\left(x_{i}^{\prime}, y_{i}^{\prime}\right) \in h, x_{i}>x_{i}^{\prime} \Leftrightarrow y_{i}>y_{i}^{\prime}$.

Lemma 1. Let $\mathcal{R} \subseteq \mathcal{R}_{0}$. A mechanism $\mu: \mathcal{R}^{N} \rightarrow Z$ satisfies $S P$ if and only if for every $i \in N$, there exists a correspondence $\mathcal{O}_{i}: \mathcal{R}^{N \backslash\{i\}} \rightarrow \mathbb{R}_{+} \times \mathbb{R}$, such that for every $R \in \mathcal{R}^{N}$,

(i) $\mu_{i}(R) \in m\left(\mathcal{O}_{i}\left(R_{-i}\right), R_{i}\right)$,

(ii) $\mathcal{O}_{i}\left(R_{-i}\right)$ is a strictly increasing subset of $\mathbb{R}_{+} \times \mathbb{R}$.

Lemma 2. Let $f: \mathbb{R}_{+} \rightarrow \mathbb{R}$ be an increasing concave function and let $g: \mathcal{D} \rightarrow \mathbb{R}$ with $\mathcal{D} \subseteq \mathbb{R}_{+}$. Let $l_{m} \geq 0$ and $x_{m}=\sup \left(\arg \max _{t \in \mathcal{D}} g(t)-l_{m} t\right)$. Then, if

$$
\forall l \in] 0, l_{m}\left[, \quad \arg \max _{t \in \mathbb{R}_{+}}(f(t)-l t) \supseteq \arg \max _{t \in \mathcal{D}}(g(t)-l t) \neq \emptyset\right.
$$

(with the convention that arg $\max { }_{t \in \mathbb{R}_{+}}(f(t)-l t)=\{+\infty\} \neq \emptyset$ if $l<\lim _{t \rightarrow \infty} f^{\prime}(t)$ ), there exists $\alpha \in \mathbb{R}$ such that $g$ and $f+\alpha$ coincide on $\mathcal{D} \cap\left[x_{m},+\infty\right]$.

Proof. Define, for any $l \in] 0, l_{m}[$,

$$
\psi(l)=\max _{t \geq 0} f(t)-l t \quad \text { and } \quad \theta(l)=\max _{t \in \mathcal{D}} g(t)-l t
$$

Letting $x(l)$ be a solution of $\max _{t \geq 0} f(t)-l t$, writing $\psi(l)=f(x(l))-l x(l)$ yields that the derivative of $\psi$ at $l$ equals $\psi^{\prime}(l)=x^{\prime}(l)\left(f^{\prime}(x(l))-l\right)-x(l)$ with either $f^{\prime}(x(l))=l$ (in general) or $x^{\prime}(l)=0$ (at a kink in the graph of $f$ ). I.e., $\psi^{\prime}(l)=-x$ for some $x \in \arg \max _{t \geq 0} f(t)-l t$. From the concavity of $f$, $\arg \max _{t \geq 0}$ $f(t)$ - lt is single-valued everywhere except on a countable subset of values of $l$, corresponding to the linear parts of $f$ (if any). Similarly, the derivative of $\theta$ at $l$ equals $\theta^{\prime}(l)=-x$ for some $x \in \arg \max _{t \in \mathcal{D}}$ $g(t)-l t$ for any $l \in] 0, l_{m}[$. 


\section{ARTICLEE IN PRESS}

From (1), $\psi^{\prime}$ and $\theta^{\prime}$ must coincide almost everywhere on $] 0, l_{m}[$. Hence, $\psi$ and $\theta$ coincide up to a constant on $] 0, l_{m}\left[\right.$. Therefore $f$ and $g$ coincide up to a constant on $\mathcal{D} \cap\left[x_{m},+\infty\right]$.

We now tackle the proof of the theorem. For the sake of contradiction let $\mu: \mathcal{L}^{N} \rightarrow Z$ satisfy SP and PE. For any $L \in \mathcal{L}^{N}$ denote the corresponding vector of slopes $\left(l_{1}, \ldots, l_{n}\right)$ and write $\mu(L)=\left(x_{i}, y_{i}\right)_{i \in \mathrm{N}}$. Define $\underline{l}=$ $\min _{j \in N} l_{j}$ and $J=\arg \min _{j \in N} l_{j}$. Denote by $F^{\prime}$ (resp. $F^{\prime}{ }_{-}$) the derivative (resp. left-derivative) of $F$. Because $F$ is non-linear, we can assume

$$
F^{\prime}(0)>\underline{l}>\lim _{t \rightarrow+\infty} F^{\prime}-(t) .
$$

\section{Step 1}

$$
\begin{cases}x_{i}>0 \text { only if } i \in J, & (\alpha) \\ x_{N}=x_{J} \in \arg \max (F(t)-\underline{l} t) & (\beta) \text { and }, \\ y_{N}=F\left(x_{N}\right) . & (\gamma)\end{cases}
$$

Condition $(\alpha)$ follows from trade efficiency: any agent $i$ for which $l_{i}>\underline{l}$ and $x_{i}>0$ would gladly pay $\frac{l_{i}+\underline{l}}{2}$ units of output in order to provide one less unit of input; any agent $j \in J$ would accept to trade with $i$. $(\beta)$ follows from $(\alpha)$ and production efficiency. Condition $(\gamma)$ states that all the output is allocated.

Fix $i \in N$ and $L_{-i} \in \mathcal{L}^{M\{i\}}$ until Step 5. We apply Lemma 1 and write $\mathcal{O}_{i}\left(L_{-\mathrm{i}}\right)$ the option set of agent $i$. Define $l_{i}^{-}=\min _{j \neq i} l_{j}$ and $\hat{x}_{i}=\max \left(\arg \max \left(F(t)-l_{i}^{-} t\right)\right)$. Notice that $\hat{x}_{i}$ is finite because $l_{i}^{-}>\lim _{t \rightarrow \infty}$ $F^{\prime}(t)$ (from (2)). For any $L_{i} \in \mathcal{L}$, denote by $l_{i} \in \mathbb{R}_{++}$the corresponding slope. We write $\mu\left(L_{i} ; L_{-i}\right)=\left(x_{i}\left(l_{i}\right)\right.$, $\left.y_{i}\left(l_{i}\right)\right)_{i \in N}$.

Steps 2-4 are devoted to the description of the shape of $\mathcal{O}_{i}\left(L_{-i}\right)$.

\section{Step 2}

$$
\mathcal{O} i\left(L_{-i}\right) \cap\left(\left[0, \hat{x}_{i}\right] \times \mathbb{R}\right) \subseteq\left\{(x, y) \in\left[0, \hat{x}_{i}\right] \times \mathbb{R} \mid y=\alpha_{i}+l_{i}^{-} x\right\} \quad \text { for some } \alpha_{i} \in \mathbb{R} .
$$

Let $l_{i}>l_{i}^{-}$, condition (3. $\left.\alpha\right)$ requires $x_{i}\left(l_{i}\right)=0$; denote $\alpha_{i}=y_{i}\left(l_{i}\right)$. We claim that $z_{i}\left(l^{\prime}{ }_{i}\right)=\left(0, \alpha_{i}\right)$ for all $l_{i}^{\prime}>l_{i}^{-}$ because of SP. Indeed, assume there existed some $l_{i}^{\prime}>l_{i}^{-}$such that $z_{i}\left(l_{i}^{\prime}\right)=\left(0, \alpha_{i}^{\prime}\right)$ with $\alpha_{i}^{\prime}, \neq \alpha_{i}$. If $\alpha^{\prime}{ }_{i}>\alpha_{i}$, agent $i$ could benefit from reporting $l_{i}^{\prime}$ at $\left(L_{i}, L_{-i}\right)$; if the inequality were reversed, agent $i$ could benefit from reporting $l_{i}$ at $\left(L_{i}^{\prime}, L_{-i}\right)$.

Now if $l_{I}=l_{i}^{-}, x_{i}\left(l_{i}^{-}\right) \in\left[0, \hat{x}_{i}\right]$ by condition $(3 . \beta)$; we show that SP requires $y_{i}\left(l_{i}^{-}\right)=\alpha_{i}+l_{i}^{-} x_{i}\left(l_{i}^{-}\right)$. Assume $y_{i}\left(l_{i}^{-}\right)>\alpha_{i}+l_{i}^{-} x_{i}\left(l_{i}^{-}\right)=0$, then $y_{i}\left(l_{i}^{-}\right)>\alpha_{i}$ and agent $i$ can benefit from reporting $l_{i}^{-}$when her true preference is in fact $l_{i}^{\prime}>l_{i}^{-}$. If $x_{i}\left(l_{i}^{-}\right)>0$, let $l_{i}^{*}=\frac{y_{i}\left(l_{i}^{-}\right)-\alpha_{i}}{x_{i}\left(l_{i}^{-}\right)}>l_{i}^{-}$; agent $i$ could benefit from reporting $l_{i}^{-}$ instead of $\left.l_{i}^{\prime} \in\right] l_{i}^{-}, l_{i}^{*}\left[\right.$. Therefore $y_{i}\left(l_{i}^{-}\right) \leq \alpha_{i}+l_{i}^{-} x_{i}\left(l_{i}^{-}\right)$. Similarly, $y_{i}\left(l_{i}^{-}\right) \geq \alpha_{i}+l_{i}^{-} x_{i}\left(l_{i}^{-}\right)$.

Also, for any $l_{i}<l_{i}^{-}, x_{i}\left(l_{i}\right) \geq \hat{x}_{i}$; and if there exists some $l_{i}^{\prime}<l_{i}^{-}$for which $x_{i}\left(l_{i}^{\prime}\right)=\hat{x}_{i}$, the same reasoning as above yields $y_{i}\left(l_{i}^{\prime}\right)=\alpha_{i}+l_{i}^{-} \hat{x}_{i}$. Step 2 has been proved.

For the next step, we use the following notation: for any $k \in \mathbb{R}$, define $B(k)=\left\{\left(x_{i}, y_{i}\right) \in \mathbb{R}_{+} \times \mathbb{R} \mid y_{i}=\right.$ $\left.F\left(x_{i}\right)+k\right\}$. 


\section{Step 3}

$\mathcal{O}_{i}\left(L_{-i}\right) \cap\left(\left[\hat{x}_{i},+\infty[\times \mathrm{R}) \subset B\left(\beta_{i}\right)\right.\right.$ for some $\beta_{i} \in \mathbb{R}$.

Let $\omega$ be the real-valued function whose graph is $\mathcal{O}_{i}\left(L_{-i}\right)$ and $\mathcal{D}$ its domain. We wish to apply Lemma 2 where $F$ plays the role of $f, \omega$ the role of $g$ and where $l_{m}=l_{i}^{-}$. We need to check that for any $0<l_{i}<l_{i}^{-}$,

$$
\emptyset \neq \arg \max _{t \in \mathcal{D}} \omega(t)-l_{i} t \subseteq \arg \max _{t \geq 0} F(t)-l_{i} t .
$$

Let $0<l_{i}<l_{i}^{-}$write $\mu_{i}(L)=\left(x_{i}, y_{i}\right)$. From Lemma $1\left(x_{i}, y_{i}\right) \in m\left(\mathcal{O}_{i}\left(L_{-i}\right), L_{i}\right)$; therefore, by the definition of $\omega, x_{i} \in \arg \max _{t \in \mathcal{D}} \omega(t)-l_{i} t$. However, $J=\{i\}$ because $l_{i}<l_{i}^{-}$; condition (3. $\left.\beta\right)$ yields $x_{i} \in \arg \max _{t} \geq 0$ $F(t)-l_{i} t$. We can apply Lemma 2 and conclude that $\omega$ and $F$ coincide up to a constant on $\left[\hat{x}_{i},+\infty[\right.$.

\section{Step 4}

$$
\beta_{i}=\alpha_{i}+l_{i}^{-} \hat{x}_{i}-F\left(\hat{x}_{i}\right)
$$

By concavity of $F$ and by definition of $\hat{x}_{i}, F$ is strictly concave at $\hat{x}_{i}$. Therefore, $\lim _{l \uparrow} l_{i}^{-}(\arg \max (F(t)$ $l t))=\hat{x}_{i}$, and hence $\lim _{l \uparrow} l_{i}^{-} x_{i}(l)=\hat{x}_{i}$. Steps 2 and 3 yield the result.

Now that the shape of $\mathcal{O}_{i}\left(L_{-i}\right)$ has been determined, we show that it is an implausible one. Note that $\alpha_{i}$ is actually a function of $L_{-i}$; from now on we write $\alpha_{i}\left(L_{-i}\right)$. For any $L \in \mathcal{L}^{N}$ let $l_{n-1}^{*}$ denote the second smallest entry of the corresponding vector of slopes $\left(l_{1}, \ldots, l_{n}\right) \in \mathbb{R}_{++}^{n}$. Also, for any $l \in \mathbb{R}_{++}$, define $h(l)=\max (F(t)-l t)$.

\section{Step 5}

For any $L \in \mathcal{L}^{N}, \sum \alpha_{i}\left(L_{-i}\right)=h\left(l_{n-1}^{*}\right)$.

Consider $L \in \mathcal{L}^{N}$ and the corresponding $\left(l_{1}, \ldots, l_{n}\right) \in \mathbb{R}_{+}^{N}$. Recall that $J=\arg \min _{j \in N} l_{j}$; clearly $J \neq \emptyset$. Step 1 requires $x_{i}=0$ and $y_{i}=\alpha_{i}\left(L_{-i}\right)$ for all $i \notin J$. If $|J|=1$, say $J=\{j\}$, then $y_{i}=\alpha_{j}\left(L_{-j}\right)+l_{n-1}^{*} \hat{x}_{j}+F\left(x_{j}\right)-F\left(\hat{x}_{j}\right)$. By efficiency:

$$
\begin{array}{ll}
y_{N}= & F\left(x_{N}\right), \\
\text { i.e. } & \sum \alpha_{i}\left(L_{-i}\right)+l_{n-1}^{*} \hat{x}_{j}+F\left(x_{j}\right)-F\left(\hat{x}_{j}\right)=F\left(x_{j}\right), \\
\text { i.e. } & \sum \alpha_{i}\left(L_{-i}\right)+h\left(l_{n-1}^{*}\right) \text { by construction of } \hat{x}_{j} .
\end{array}
$$

Now suppose $|J| \geq 2$, then $l_{n-1}^{*}=\underline{l}$. Moreover, for any $j \in J, x_{j} \geq 0, y_{j}=\alpha_{j}\left(L_{-j}\right)+l_{n-1}^{*} x_{j}$ and $x_{J} \in \arg$ $\left.\max F(t)-l_{n-1}^{*} t\right)$. Once again the result follows from efficiency:

$$
\begin{aligned}
& y_{N}=F\left(x_{N}\right), \\
& \text { i.e. } \quad \sum \alpha_{i}\left(L_{-i}\right)+l_{n-1}^{*} x_{J}=F\left(x_{J}\right), \\
& \text { i.e. } \quad \sum \alpha_{i}\left(L_{-i}\right)=h\left(l_{n-1}^{*}\right) .
\end{aligned}
$$


Upon noticing that $h$ is strictly decreasing on $] \underline{l}, F^{\prime}(0)[$, a slight variation of a standard argument in the literature on Clarke-Grove mechanisms (omitted for brevity but available upon request) yields a contradiction: $\left(l_{1}, \ldots, l_{n}\right) \mapsto h\left(l_{n-1}^{*}\right)$ cannot be decomposed into $n$ functions depending only on $n-1$ variables.

Remark. We strongly suspect that our proof technique successfully applies to the many-inputs-oneoutput case without many conceptual modifications.

\section{Acknowledgements}

I am grateful to Hervé Moulin for his patience and valuable advice. This work has also benefited from conversations with James Schummer and Lin Zhou.

\section{References}

Hurwicz, L., 1972. On informationally decentralized systems. In: McGuire, C.B., Radner, R. (Eds.), Decision and Organization. North-Holland, Amsterdam, pp. 297-336.

Ju, B.-G., 2003. Strategy-proofness versus efficiency in exchange economies: general domain properties and applications. Social Choice and Welfare 21, 73-93.

Kato, M., Ohseto, S., 2002. Toward general impossibility theorems in pure exchange economies. Social Choice and Welfare 19, 659-664.

Maniquet, F., Sprumont, Y., 1999. Efficient strategy-proof allocation functions in linear production economies. Economic Theory $14,583-595$.

Schummer, J., 1997. Strategy-proofness versus efficiency on restricted domains of exchange economies. Social Choice and Welfare 14, 47-56.

Serizawa, S., 2002. Inefficiency of strategy-proof rules for pure exchange economies. Journal of Economic Theory 106, 219-241.

Shenker, S., 1992, On the Strategy-Proof and Smooth Allocation of Private Goods in a Production Economy Mimeo, Xerox PARC.

Sprumont, Y., 1995. A note on strategy-proofness in Edgeworth-Box economies. Economic Letters 49, 45-50.

Zhou, L., 1991. Inefficiency of strategy-proof allocation mechanisms in pure exchange economies. Social Choice and Welfare 8, 247-254. 